\title{
Lines Avoiding Unit Balls in Three Dimensions*
}

\author{
Pankaj K. Agarwal, ${ }^{1}$ Boris Aronov, ${ }^{2}$ Vladlen Koltun, ${ }^{3}$ and Micha Sharir ${ }^{4}$ \\ ${ }^{1}$ Department of Computer Science, Duke University, \\ Durham, NC 27708-0129, USA \\ pankaj@cs.duke.edu \\ ${ }^{2}$ Department of Computer and Information Science, Polytechnic University, \\ Brooklyn, NY 11201-3840, USA \\ aronov@cis.poly.edu \\ ${ }^{3}$ Computer Science Division, University of California, \\ Berkeley, CA 94720-1776, USA \\ vladlen@cs.berkeley.edu \\ ${ }^{4}$ School of Computer Science, Tel Aviv University, \\ Tel Aviv 69978, Israel \\ michas@post.tau.ac.il \\ and \\ Courant Institute of Mathematical Sciences, New York University, \\ New York, NY 10012, USA
}

\begin{abstract}
Let $\mathcal{B}$ be a set of $n$ unit balls in $\mathbb{R}^{3}$. We show that the combinatorial complexity of the space of lines in $\mathbb{R}^{3}$ that avoid all the balls of $\mathcal{B}$ is $O\left(n^{3+\varepsilon}\right)$, for any $\varepsilon>0$. This result has connections to problems in visibility, ray shooting, motion planning, and geometric optimization.
\end{abstract}

\section{Introduction}

Let $\mathcal{B}$ be a set of $n$ unit balls in $\mathbb{R}^{3}$. A line in $\mathbb{R}^{3}$ is called free (with respect to $\mathcal{B}$ ) if it does not meet the interior of any ball in $\mathcal{B}$; we also say that the line avoids all the balls

* Work on this paper has been supported by a joint grant from the U.S.-Israel Binational Science Foundation. Work by Pankaj Agarwal was also supported by NSF under Grants CCR-00-86013, EIA-98-70724, EIA-99-72879, EIA-01-31905, and CCR-02-04118. Work by Boris Aronov was also supported by NSF Grants CCR-99-72568 and ITR CCR-00-81964. Work by Vladlen Koltun was also supported by NSF Grant CCR01-21555. Work by Micha Sharir was also supported by NSF Grants CCR-97-32101 and CCR-00-98246, by a grant from the Israeli Academy of Sciences for a Center of Excellence in Geometric Computing at Tel Aviv University, and by the Hermann Minkowski-MINERVA Center for Geometry at Tel Aviv University. 
of $\mathcal{B}$. Let $\mathcal{F}=\mathcal{F}(\mathcal{B})$ denote the space of lines that are free with respect to $\mathcal{B}$. We regard $\mathcal{F}$ as a subset of some four-dimensional parametric space $\mathbb{L}$ that represents lines in $\mathbb{R}^{3}$ [8]. In this paper we study the combinatorial complexity of $\mathcal{F}$, which, for the time being, we define as the number of lines in $\mathcal{F}$ that are tangent to four balls in $\mathcal{B}$. These are the "vertices" of $\mathcal{F}$; see below for a more precise definition.

The space $\mathcal{F}$ is, in a sense, "antithetical" to the space $\mathcal{T}=\mathcal{T}(\mathcal{B})$ of line transversals of $\mathcal{B}$, i.e., the space of lines that intersect all the balls of $\mathcal{B}$. It is known (see, e.g., [13]) that, with an appropriate choice of $\mathbb{L}, \mathcal{T}$ can be represented as a sandwich region lying between the upper envelope of one family of surfaces in $\mathbb{L}$, consisting of the loci of lines tangent to the balls of $\mathcal{B}$ from below, and the lower envelope of another such family, consisting of the loci of lines tangent to the balls of $\mathcal{B}$ from above. The recent result of Koltun and Sharir [13] immediately yields a near-cubic bound on the complexity of $\mathcal{T}$. However, the space $\mathcal{F}$ of free lines does not appear to admit such a representation, and the best previously known upper bound for its complexity was the trivial bound of $O\left(n^{4}\right)$, obtained by observing that $\mathcal{F}$ is the union of certain cells of the arrangement of the aforementioned tangent-line surfaces in $\mathbb{L}$, and that the complexity of this entire four-dimensional arrangement is at most $O\left(n^{4}\right)$. The following theorem is the main result of this paper.

Theorem 1.1. The combinatorial complexity of the space of lines free with respect to a set of $n$ unit balls in $\mathbb{R}^{3}$ is $O\left(n^{3+\varepsilon}\right)$, for any $\varepsilon>0$.

This result is related to several topics of study in computational and combinatorial geometry. For instance, the structure and complexity of the space of free lines is intimately connected to the analysis of global-visibility data structures [10], [16], whose size crucially depends on the complexity of the space of free segments amid the given objects (defined in analogy to the space of free lines). Our result is also closely related to robot motion planning - the space $\mathcal{F}(\mathcal{B})$ is the free configuration space [17] of a line robot moving amid unit ball obstacles in $\mathbb{R}^{3}$, or, alternatively, of a cylindrical robot (of infinite length) moving amid point obstacles or amid congruent balls. Finally, our result is related to the problem of computing the largest empty cylinder amid $n$ points in $\mathbb{R}^{3}$, to ray shooting, and to several other problems in geometric optimization.

The derivation of Theorem 1.1 requires a number of novel technical contributions. Our conceptual starting point is the near-cubic bound on the complexity of the sandwich region lying between envelopes of two families of surfaces in $\mathbb{R}^{4}$ [13]. As mentioned above, the space $\mathcal{F}$ cannot be directly represented as such a sandwich region. We present a series of reductions that allow us to bound the complexity of $\mathcal{F}$ in terms of the cumulative complexity of a collection of appropriately defined sandwich regions. The number of these sandwich regions and of the surfaces defining them may however be unbounded. To achieve the desired near-cubic bound we introduce a refinement of the bound of [13] for sandwich regions, which shows that it is nearly proportional to the number of triples of surfaces whose domains overlap, as defined below. This refinement appears to be of independent interest.

Related Work. The complexity of the space of free lines amid various types of polyhedral objects has been considered in the past, with mixed success. When the objects 
constitute a polyhedral region of total complexity $n$, or even in the special case of a collection of $n$ lines in $\mathbb{R}^{3}$, the maximum complexity of the space of free lines is easily seen to be $\Theta\left(n^{4}\right)$ [8]. However, the complexity of the space of lines that avoid a polyhedral terrain having $n$ faces is only near-cubic [15]. The $\Omega\left(n^{4}\right)$ lower bound constructions for the cases of polyhedra and lines are rather unrealistic, and the hope is that, when the objects are fat or well distributed, the complexity of the space of free lines is reduced. Collections of unit balls are the simplest example of such a well-behaved class of objects, which can still model fully three-dimensional scenes, and they have indeed already been studied in this context [9], [10]. A related result [5] obtains a near-cubic bound on the complexity of the set of lines free relative to a collection of $n$ homothets of a bounded-complexity convex polyhedron.

To calibrate the result of this paper and to put it in a wider context, we note that lines in $\mathbb{R}^{3}$ have four degrees of freedom, and can therefore be represented as points in $\mathbb{R}^{4}$ (the space $\mathbb{L}$ alluded to above). ${ }^{1}$ Hence, $\mathcal{F}(\mathcal{B})$ is a subregion of $\mathbb{R}^{4}$. In fact, if we consider $n$ surfaces in $\mathbb{R}^{4}$, each being the locus of all (points representing) lines tangent to a fixed ball in $\mathcal{B}$, and form the arrangement of these surfaces, then each cell of the arrangement consists of lines that intersect all the balls in a fixed subset of $\mathcal{B}$ and avoid the remaining balls. This arrangement has $O\left(n^{4}\right)$ cells of all dimensions, and $\mathcal{F}(\mathcal{B})$ is the union of a subset of them. Hence, the complexity of $\mathcal{F}(\mathcal{B})$ is $O\left(n^{4}\right)$, and it can be trivially constructed in near-quartic time. (In fact, these properties hold for any collection $\mathcal{B}$ of $n$ objects of simple shape in $\mathbb{R}^{3}$.) A near-cubic upper bound for the case of unit balls is thus an improvement by nearly a factor of $n$ over the above general observation.

Another way of looking at this problem is to define, for each ball $B \in \mathcal{B}$, the region $K_{B}$, consisting of all (points representing) lines that intersect $B$ (the boundary of $K_{B}$ is the aforementioned surface of lines tangent to $B$ ). Then $\mathcal{F}(\mathcal{B})$ is the complement of the union of the sets $K_{B}$, over $B \in \mathcal{B}$. Thus, analyzing the complexity of $\mathcal{F}(\mathcal{B})$ is an instance of the general study of the complexity of the union of geometric objects, an area that has received considerable attention in recent years; see [1], [3], [4], [7], [11], [12], and [14]. Our result is among the very first nontrivial bounds on the complexity of the union of geometric objects in four dimensions (excluding the significantly easier cases of halfspaces, balls, and axis-parallel hypercubes). A companion work by the authors [2] derives a near-cubic bound for the union of $\kappa$-round objects in four dimensions, extending an earlier result by Koltun and Sharir [13] (see also [1]). These are the only results of this type of which we are aware.

Proof Outline. Our analysis of the complexity of $\mathcal{F}(\mathcal{B})$ proceeds through a number of steps, each constituting a further reduction of the problem. We begin, in Section 2, by defining the space $\mathcal{F}$ of free lines as a subset of an arrangement of surfaces in $\mathbb{R}^{4}$, and show that in order to bound the complexity of $\mathcal{F}$ it is sufficient to bound the number of vertices of this arrangement that lie on the boundary of $\mathcal{F}$. These vertices correspond to free lines in $\mathbb{R}^{3}$ that are tangent to quadruples of balls in $\mathcal{B}$.

\footnotetext{
${ }^{1}$ Strictly speaking, the space of lines in $\mathbb{R}^{3}$ is the so-called affine Grassmannian, which is a fourdimensional manifold not homeomorphic to $\mathbb{R}^{4}$. However, by leaving out the three-dimensional subspace of horizontal lines, we can map the space of nonhorizontal lines homeomorphically to $\mathbb{R}^{4}$. It is sufficient to work with the latter representation, since a generic choice of the coordinate system ensures that all vertices of $\mathcal{F}$ correspond to nonhorizontal lines.
} 
We then define, still in Section 2, the notion of vertices that are deep with respect to a reference direction in $\mathbb{S}^{2}$. Roughly speaking, a vertex that represents a line $\ell$ is deep with respect to a direction $u$ if, when we enter each of the four balls $B$ tangent to $\ell$ from the respective point of tangency in the direction orthogonal to both $u$ and $\ell$, we traverse a "long" chord of $B$. It is shown that there exists a reference direction for which at least half of the vertices are deep. Hence it suffices to bound the maximum number of vertices that are deep with respect to some fixed reference direction, a task that is undertaken in Section 3. We bound the number of deep vertices by deriving a recurrence relation on this quantity. We project the centers of the balls in $\mathcal{B}$ onto the $x y$-plane, and partition the projected centers using a (nonuniform) planar grid, such that every row and every column contains $n / r$ centers, for a certain parameter $r$. We concentrate on vertices defined by quadruples of balls whose four centers project into four distinct rows and four distinct columns, and use recursion to bound the number of other types of vertices. We fix a quadruple of grid cells that lie in distinct rows and columns, and argue that the four corresponding sets of projected ball centers are doubly well-separated with respect to some "middle" point $o$, meaning intuitively that $o$ lies "in between" one pair of cells and also in between the complementary pair of cells.

The main technical step of the proof, given in Section 4, concentrates on proving a near-cubic bound on the number of deep vertices defined by a doubly well-separated quadruple of sets of balls. By carefully selecting a coordinate frame for the fourdimensional space of lines, we are able to express, in Section 4.1, each such vertex as a vertex in one of a number of sandwich regions of envelopes of four-dimensional arrangements that we define. This is where we exploit the nature of deep vertices and the fact that the lines that we are considering are free. Despite the existence of a good (i.e., near-cubic) bound on the complexity of sandwich regions in four dimensions [13], this is not the end of the story yet, since the number of the resulting sandwich regions, as well as the overall size of all the sets of surfaces that form these regions, may be unbounded. In Section 4.2 we show that the functions determining the sandwich regions have sparse domains, in a certain sense defined in that section. This is where we exploit the nature of doubly well-separated sets and the fact that the balls are congruent. In Section 5 we establish a refined bound on the complexity of sandwich regions of envelopes of arrangements of trivariate functions with sparse domains, a result that we believe to be of independent interest. Using this result, we are able to prove the desired nearcubic bound on the number of deep vertices defined by doubly well-separated sets of balls.

Plugging this bound into the analysis of Section 3, we obtain the desired recurrence for the number of deep vertices, which solves to a near-cubic bound. Due to the reductions mentioned above, this bound leads directly to the main result of the paper.

\section{Reduction to Deep Vertices}

Let $\mathcal{B}=\left\{B_{1}, \ldots, B_{n}\right\}$ be a set of $n$ unit balls in $\mathbb{R}^{3}$. We assume that the balls in $\mathcal{B}$ are in general position, meaning that no line is tangent to any five distinct balls and that only a finite number of lines are tangent to any four balls. This involves no loss of generality because we can apply a sufficiently small random perturbation to the balls, putting them 


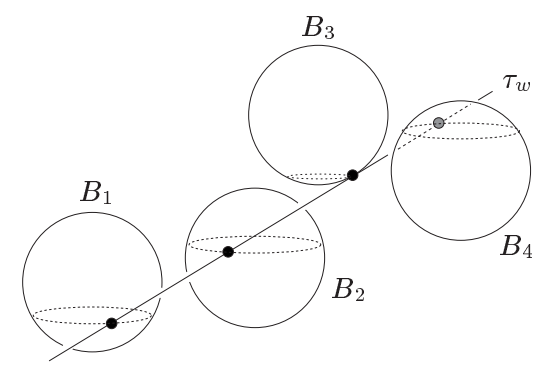

Fig. 1. The illustrated line $\tau_{w}$ is represented by a vertex $w$ of $\mathcal{F}(\mathcal{B})$.

in general position, without losing more than an (expected) constant fraction of the number of faces of $\partial \mathcal{F}(\mathcal{B})$; see Section 7.3.1 of [18] for similar arguments.

Let $\mathbb{L}$ be the space of all lines in $\mathbb{R}^{3}$. Since lines in $\mathbb{R}^{3}$ can be parameterized by four parameters, $\mathbb{L}$ can be regarded as a four-dimensional (real) parametric space. We recall the notation introduced above: For a ball $B \in \mathcal{B}$, let $K_{B} \subseteq \mathbb{L}$ denote the set of lines that intersect $B ; \partial K_{B}$ is the set of all lines that are tangent to $B$. By our general-position assumption, $\mathcal{F}=\mathcal{F}(\mathcal{B})=\operatorname{cl}\left(\mathbb{L} \backslash \bigcup_{B \in \mathcal{B}} K_{B}\right)$, where $\operatorname{cl}(\cdot)$ denotes closure. The boundary of $\mathcal{F}$, denoted as $\partial \mathcal{F}$, thus consists of portions of the boundaries $\partial K_{B}$, for $B \in \mathcal{B}$. Any such connected portion is in fact a face of the arrangement $\mathcal{A}$ of the boundaries $\partial K_{B}$, for $B \in \mathcal{B}$. The combinatorial complexity of $\mathcal{F}$ is the number of faces of $\mathcal{A}$ of all dimensions that appear on $\partial \mathcal{F}$. A vertex $w$ of $\mathcal{F}$ represents a free line $\tau_{w}$ in $\mathbb{R}^{3}$ that is tangent to four balls of $\mathcal{B}$; see Fig. 1 . We denote by $v_{w} \in \mathbb{S}^{2}$ the orientation ${ }^{2}$ of $\tau_{w}$, and by $\mathcal{B}_{w} \subseteq \mathcal{B}$ the set of four balls tangent to $\tau_{w}$. Let $\mathcal{W}=\mathcal{W}(\mathcal{B})$ denote the set of vertices of $\mathcal{F}$.

Lemma 2.1. For a set $\mathcal{B}$ of $n$ unit balls in $\mathbb{R}^{3}$, the combinatorial complexity of $\mathcal{F}$ is $O\left(|\mathcal{W}|+n^{3}\right)$.

Proof. Any face of $\partial \mathcal{F}$ that is incident upon a vertex of $\mathcal{F}$ can be charged to any of its vertices. The general-position assumption implies that each vertex is charged in this manner at most a constant number of times, and the number of such faces is thus $O(|\mathcal{W}|)$. A face $f$ that is not incident upon a vertex of $\mathcal{F}$ is defined by at most three balls $B_{1}, \ldots, B_{l} \in \mathcal{B}$, where $l \in\{1,2,3\}$, and either coincides with, or is incident upon, an entire connected component of $\bigcap_{i=1}^{l} \partial K_{B_{i}}$. We can thus charge $f$ to such a component, and observe that, as above, the general position assumption implies that no component is charged more than $O(1)$ times. Since any intersection $\bigcap_{i=1}^{l} \partial K_{B_{i}}$ has only $O(1)$ connected components, the total number of faces that are not incident upon a vertex is $O\left(n^{3}\right)$.

Fix a reference direction $u$ in $\mathbb{S}^{2}$. Let $w$ be a vertex in $\mathcal{W}$, and let $v_{w} \neq u$ be the orientation of $\tau_{w}$. Let $B_{1}, B_{2}, B_{3}, B_{4}$ be the balls in $\mathcal{B}_{w}$, and let $\ell_{i}$ be the line passing

\footnotetext{
${ }^{2}$ Strictly speaking, the lines in $\mathbb{L}$ are not considered to be oriented, so $v_{w}$ is encoded by two antipodal points on $\mathbb{S}^{2}$. We ignore this technicality for the sake of simplicity.
} 


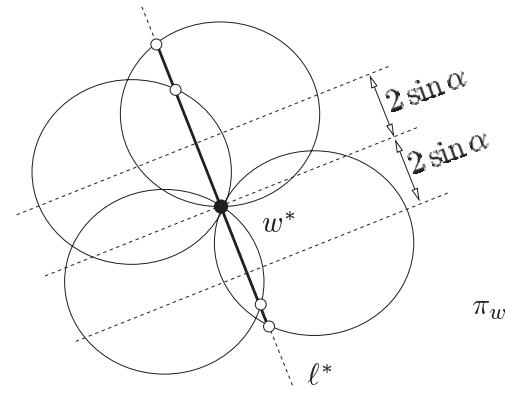

Fig. 2. A deep vertex $w$ of $\mathcal{F}$ and the associated projected configuration in the plane $\pi_{w}$ normal to $\tau_{w}$.

through the tangency point $\tau_{w} \cap B_{i}$ and orthogonal to both $u$ and $v_{w}$; these four lines are parallel. We call $w$ deep (with respect to $u$ ) if for each $i \in\{1,2,3,4\}$, the length of the chord $\ell_{i} \cap B_{i}$ is at least $2 \sin \alpha$ (or, equivalently, the central angle that the chord subtends is at least $2 \alpha$ ), where $\alpha$ is a constant to be fixed below. Project $\tau_{w}$ and the balls in $\mathcal{B}_{w}$ onto a plane $\pi_{w}$ normal to $\tau_{w}$. Then $\tau_{w}$ projects to a point $w^{*}$ and the four balls $B_{1}, \ldots, B_{4}$ project to four unit disks $B_{1}^{*}, \ldots, B_{4}^{*}$ whose boundaries all pass through $w^{*}$.

The four lines $\ell_{i}$ project to a common line $\ell^{*}$ that passes through $w^{*}$. If $w$ is deep, then the length of each of the chords $\ell^{*} \cap B_{i}^{*}$ is at least $2 \sin \alpha$; see Fig. 2.

Lemma 2.2. There exists a reference direction $u \in \mathbb{S}^{2}$ such that the number of vertices in $\mathcal{W}$ that are deep with respect to $u$ is at least $|\mathcal{W}| / 2$, provided that $\alpha \leq \pi / 16$.

Proof. We will show that if $\alpha \leq \pi / 16$ and $u$ is chosen randomly and uniformly from $\mathbb{S}^{2}$, then the expected number of vertices in $\mathcal{W}$ that are deep with respect to $u$ is at least $|\mathcal{W}| / 2$. This implies in particular that there must exist a direction $u$ for which the property described in the lemma holds.

Let $w \in \mathcal{W}$, let $v:=v_{w}$ be the orientation of $\tau:=\tau_{w}$, and let $\mathcal{B}_{w}=\left\{B_{1}, B_{2}, B_{3}, B_{4}\right\}$ be the set of balls tangent to $\tau$. Fix one of these balls, say $B:=B_{1}$. Without loss of generality, assume that $\tau$ is the $z$-axis, and that $B$ is the unit ball centered at $(1,0,0)$. Regard the direction $u$ as $b a d$ (for $B$ ) if the horizontal line $\ell$ in direction $u \times v$ through the origin intersects $B$ in a chord whose length is smaller than $2 \sin \alpha$. Clearly, if $u$ is bad then $\ell$ meets (the horizontal cross section of) $B$ in a chord that subtends a central angle of less than $2 \alpha$, or, equivalently, the direction $u \times v$ of $\ell$ forms an angle less than $\alpha$ with the $y$-axis. See Fig. 3(i),(ii). Therefore, $u \in \mathbb{S}^{2}$, being orthogonal to $u \times v$, must lie in a spherical double wedge of opening angle $2 \alpha$ centered around the great circle of $\mathbb{S}^{2}$ normal to the $y$-axis. See Fig. 3(iii). Thus a random choice of $u$ would land in this double wedge with probability $2 \alpha / \pi$.

Applying this analysis to all four balls $B_{1}, \ldots, B_{4}$, we conclude that the probability that a random direction $u$ is bad relative to at least one ball is no more than $8 \alpha / \pi$, and setting $\alpha$ to any value not exceeding $\pi / 16$ assures that a random choice of $u$ is good for $w$ with probability at least $1 / 2$, i.e., with probability at least $1 / 2, w$ is a deep vertex with respect to $u$. This completes the proof. 


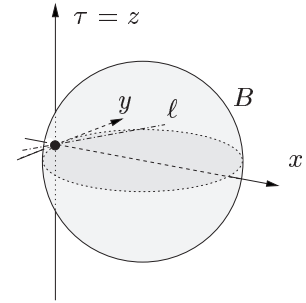

(i)

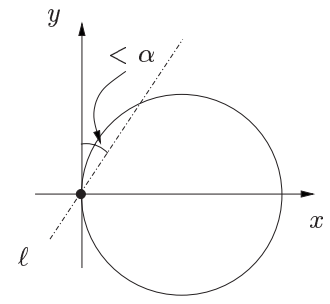

(ii)

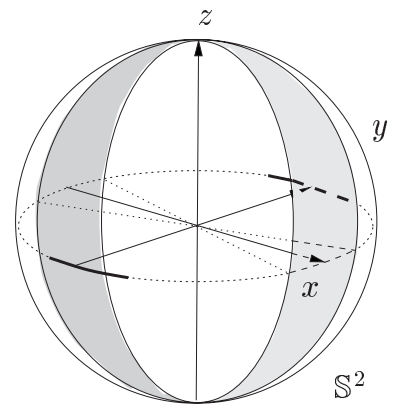

(iii)

Fig. 3. Illustrating the proof of Lemma 2.2. (i) The line $\ell$ and the ball $B$. (ii) The cross section of (i) by the $x y$-plane. (iii) The double wedge of bad directions on $\mathbb{S}^{2}$.

\section{Counting Deep Vertices}

In view of Lemma 2.2, it suffices to bound the number of vertices in $\mathcal{W}$ that are deep with respect to some fixed direction $u$, which, without loss of generality, we may assume to be the $(+z)$-direction. For a subset $\mathcal{R} \subseteq \mathcal{B}$, let $\mathcal{D}(\mathcal{R}) \subseteq \mathcal{W}(\mathcal{R})$ denote the set of vertices that are deep with respect to the vertical direction; set $\psi(\mathcal{R}):=|\mathcal{D}(\mathcal{R})|$ and

$$
\psi(m):=\max _{\substack{\mathcal{R} \subset \mathcal{B} \\|\mathcal{R}|=m}} \psi(\mathcal{R}) .
$$

We derive a recurrence relation for $\psi(m)$.

Let $c_{i}^{*}$ be the $x y$-projection of the center $c_{i}$ of $B_{i}$, and let $C^{*}=\left\{c_{1}^{*}, \ldots, c_{n}^{*}\right\}$ be the set of the $n$ projected centers in $\mathbb{R}^{2}$. Fix a parameter $r$, and partition $C^{*}$ into $r$ subsets of equal cardinality $n / r$ by $r-1$ lines $x=a_{1}, \ldots, x=a_{r-1}$ lying in the $x y$-plane parallel to the $y$-axis. We assume for simplicity and without loss of generality that $n$ is integrally divisible by $r$. Construct a similar partition of $C^{*}$ into $r$ subsets of equal cardinality by $r-1$ lines $y=b_{1}, \ldots, y=b_{r-1}$ lying in the $x y$-plane parallel to the $x$-axis. These $2(r-1)$ lines partition the $x y$-plane into the $r \times r$ nonuniform grid

$$
\mathcal{G}:=\left\{\kappa_{i j}:=\left[a_{i-1}, a_{i}\right] \times\left[b_{j-1}, b_{j}\right] \mid i, j=1, \ldots, r\right\},
$$

where we put $a_{0}, b_{0}:=-\infty$ and $a_{r}, b_{r}:=+\infty$. For $i, j=1, \ldots, r$, let $\mathcal{B}_{i j}:=\left\{B_{l} \mid\right.$ $\left.c_{l}^{*} \in \kappa_{i j}\right\}$; set $n_{i j}:=\left|\mathcal{B}_{i j}\right|$. We have $\sum_{i} n_{i j}=n / r$ for any fixed $j$ and $\sum_{j} n_{i j}=n / r$ for any fixed $i$. Let $w$ be a deep vertex in $\mathcal{D}(\mathcal{B})$, let $c_{1}^{*}, c_{2}^{*}, c_{3}^{*}$, and $c_{4}^{*}$ be the projections of the centers of the four respective balls $B_{1}, B_{2}, B_{3}, B_{4} \in \mathcal{B}_{w}$, and let $\kappa_{i_{1} j_{1}}, \ldots, \kappa_{i_{4} j_{4}}$ denote the four (not necessarily distinct) cells of $\mathcal{G}$ that contain $c_{1}^{*}, \ldots, c_{4}^{*}$, respectively.

Suppose first that at least two of these cells lie in the same row or in the same column of $\mathcal{G}$. We estimate the number of such vertices $w$ recursively, by solving $2\left(\begin{array}{c}r \\ 3\end{array}\right)$ subproblems, each involving the balls whose projected centers lie in any fixed triple of rows or columns of $\mathcal{G}$. The number of balls in each subproblem is at most $3 n / r$. The contribution of such vertices to $\psi(n)$ is at most $2\left(\begin{array}{l}r \\ 3\end{array}\right) \psi(3 n / r)$.

Otherwise, let $\mathcal{D}\left(\mathcal{B}_{i_{1} j_{1}}, \mathcal{B}_{i_{2} j_{2}}, \mathcal{B}_{i_{3} j_{3}}, \mathcal{B}_{i_{4} j_{4}}\right) \subseteq \mathcal{D}(\mathcal{B})$ denote the set of vertices $w$ for which the four centers of the balls in $\mathcal{B}_{w}$ project into cells $\kappa_{i_{1} j_{1}}, \kappa_{i_{2} j_{2}}, \kappa_{i_{3} j_{3}}$, and $\kappa_{i_{4} j_{4}}$ 
of $\mathcal{G}$, lying in four distinct rows $i_{1}, i_{2}, i_{3}, i_{4}$ and in four distinct columns $j_{1}, j_{2}, j_{3}, j_{4}$, respectively. Then

$$
\psi(n) \leq 2\left(\begin{array}{l}
r \\
3
\end{array}\right) \psi\left(\frac{3 n}{r}\right)+\sum\left|\mathcal{D}\left(\mathcal{B}_{i_{1} j_{1}}, \mathcal{B}_{i_{2} j_{2}}, \mathcal{B}_{i_{3} j_{3}}, \mathcal{B}_{i_{4} j_{4}}\right)\right|
$$

where the summation is over all (unordered) quadruples of cells of $\mathcal{G}$ that lie in distinct rows and in distinct columns.

We next introduce the notion of well-separated pairs of balls. We call two balls $B_{1}$ and $B_{2}$, with respective projected centers $c_{1}^{*}, c_{2}^{*}$, well-separated with respect to a point $o \in \mathbb{R}^{2}$ if

$$
\left|o c_{1}^{*}\right|,\left|o c_{2}^{*}\right| \leq\left|c_{1}^{*} c_{2}^{*}\right| .
$$

We call a set of four balls doubly well-separated with respect to $o$ if it is the union of two complementary pairs, both of which are well-separated with respect to $o$. We extend this definition by saying that four sets $\mathcal{B}_{1}, \mathcal{B}_{2}, \mathcal{B}_{3}, \mathcal{B}_{4}$ of balls are doubly well-separated with respect to $o$ if there exist two complementary pairs of sets, say $\mathcal{B}_{1}, \mathcal{B}_{2}$ and $\mathcal{B}_{3}, \mathcal{B}_{4}$, such that any pair of balls in $\mathcal{B}_{1} \times \mathcal{B}_{2}$ is well-separated with respect to $o$, and so is any pair of balls in $\mathcal{B}_{3} \times \mathcal{B}_{4}$.

Lemma 3.1 (Separation Lemma). Given four distinct rows $i_{1}, i_{2}, i_{3}, i_{4}$ and four distinct columns $j_{1}, j_{2}, j_{3}, j_{4}$, there exists a point o so that the four sets $\mathcal{B}_{i_{1} j_{1}}, \mathcal{B}_{i_{2} j_{2}}, \mathcal{B}_{i_{3} j_{3}}$, and $\mathcal{B}_{i_{4} j_{4}}$ are doubly well-separated with respect to $o$.

Proof. Draw an $x$-parallel line $\lambda_{1}$ so that two of the cells $\kappa_{i_{1} j_{1}}, \kappa_{i_{2} j_{2}}, \kappa_{i_{3} j_{3}}, \kappa_{i_{4} j_{4}}$ lie below it and two lie above. Similarly, draw a $y$-parallel line $\lambda_{2}$ that has two of the cells on either side. Let $o$ be the intersection point of $\lambda_{1}$ and $\lambda_{2}$. Clearly, either each of the four quadrants determined by $\lambda_{1}$ and $\lambda_{2}$ contains exactly one of the four cells, or two opposite quadrants each contain two of these cells and the two other quadrants do not contain any cell. In either case, there exist two complementary pairs of cells, so that the two cells in each pair lie in opposite quadrants; refer to Fig. 4.

Hence, if $\xi_{1}$ and $\xi_{2}$ are any two points, one from each of the two opposite cells in a

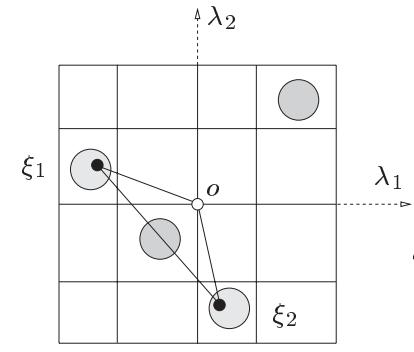

(i)

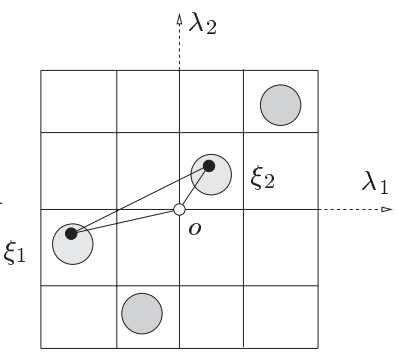

(ii)

Fig. 4. Illustrating the proof of the Separation Lemma: (i) The cells lie in four distinct quadrants. (ii) The cells lie in two opposite quadrants. The shaded disks represent the sets $\mathcal{B}_{i_{k} j_{k}}$ of projected centers. The lighter pairs of sets are well-separated with respect to $o$, and so are the darker pairs. 
pair, then the angle $\angle \xi_{1} o \xi_{2}$ is at least $\pi / 2$, implying that $\left|\xi_{1} \xi_{2}\right| \geq\left|\xi_{1} o\right|,\left|\xi_{2} o\right|$, and thus showing that any pair of balls whose centers project into these two cells is well-separated with respect to $o$. This completes the proof of the lemma.

Let $\mathcal{B}_{1}, \mathcal{B}_{2}, \mathcal{B}_{3}$, and $\mathcal{B}_{4}$ be four sets of unit balls that are doubly well-separated with respect to some point $o \in \mathbb{R}^{2}$. In the following section (see Lemma 4.5) we prove that the number of "rainbow" vertices in $\mathcal{D}\left(\mathcal{B}_{1} \cup \mathcal{B}_{2} \cup \mathcal{B}_{3} \cup \mathcal{B}_{4}\right)$, i.e., vertices $w$ such that $\mathcal{B}_{w}$ contains one ball of each of the sets $\mathcal{B}_{1}, \ldots, \mathcal{B}_{4}$, is $O\left(\left|\mathcal{B}_{1} \cup \mathcal{B}_{2} \cup \mathcal{B}_{3} \cup \mathcal{B}_{4}\right|^{3+\varepsilon}\right)$, for any $\varepsilon>0$. We apply this bound to each of the $O\left(r^{8}\right)$ quadruples of cells of $\mathcal{G}$ that lie in four distinct rows and in four distinct columns, and observe that for each such quadruple the corresponding size $\left|\mathcal{B}_{1} \cup \mathcal{B}_{2} \cup \mathcal{B}_{3} \cup \mathcal{B}_{4}\right|$ is at most $4 n / r$, to conclude that these cells contribute a total of

$$
O\left(r^{8} \cdot\left(\frac{4 n}{r}\right)^{3+\varepsilon}\right)=O\left(r^{5} n^{3+\varepsilon}\right)
$$

to $\psi(n)$. Thus the recurrence (1) for $\psi(\cdot)$ becomes

$$
\psi(n) \leq \frac{r^{3}}{3} \psi\left(\frac{3 n}{r}\right)+C r^{5} n^{3+\varepsilon},
$$

for any $\varepsilon>0$ and for a constant $C=C(\varepsilon)$. The solution of the recurrence is $\psi(n) \leq$ $A n^{3+\varepsilon}$, where the constant $A$ depends on $\varepsilon$ and $C$. Indeed, substituting into the recurrence we obtain

$$
\begin{aligned}
\psi(n) & \leq \frac{r^{3}}{3} A\left(\frac{3 n}{r}\right)^{3+\varepsilon}+C r^{5} n^{3+\varepsilon} \\
& \leq A n^{3+\varepsilon}\left(\frac{3^{2+\varepsilon}}{r^{\varepsilon}}+\frac{C r^{5}}{A}\right) \\
& \leq A n^{3+\varepsilon}
\end{aligned}
$$

provided that $r$ is chosen to be greater than $3^{(2+\varepsilon) / \varepsilon}$ and $A$ to be sufficiently large. Together with Lemmas 2.1 and 2.2, this proves Theorem 1.1, the main result of the paper.

\section{Counting Rainbow Vertices in Doubly Well-Separated Sets}

\subsection{Reduction to Sandwich Regions}

Let $\mathcal{B}_{1}, \mathcal{B}_{2}, \mathcal{B}_{3}, \mathcal{B}_{4}$ be four pairwise-disjoint sets of unit balls in $\mathbb{R}^{3}$ that are doubly wellseparated with respect to the origin, say. Set $\mathcal{B}:=\mathcal{B}_{1} \cup \mathcal{B}_{2} \cup \mathcal{B}_{3} \cup \mathcal{B}_{4}$. We call a subset $\mathcal{R} \subseteq \mathcal{B}$ of size at most four rainbow if each ball in $\mathcal{R}$ belongs to a different set $\mathcal{B}_{j}$. A vertex $w$ of $\mathcal{F}(\mathcal{B})$ is rainbow if $\mathcal{B}_{w}$ is rainbow. We wish to bound the number of rainbow vertices in $\mathcal{D}(\mathcal{B})$; this is the missing step in the proof of Theorem 1.1.

We begin by specifying our choice of the four parameters $(\theta, \varphi, \xi, \eta)$ that represent a line $\ell$ in $\mathbb{R}^{3}$. The first two parameters $(\theta, \varphi)$ are the spherical coordinates of the direction $v$ of $\ell$, where $\varphi$ is its azimuth, i.e., the angle it forms with the positive $z$-direction, and $\theta$ 


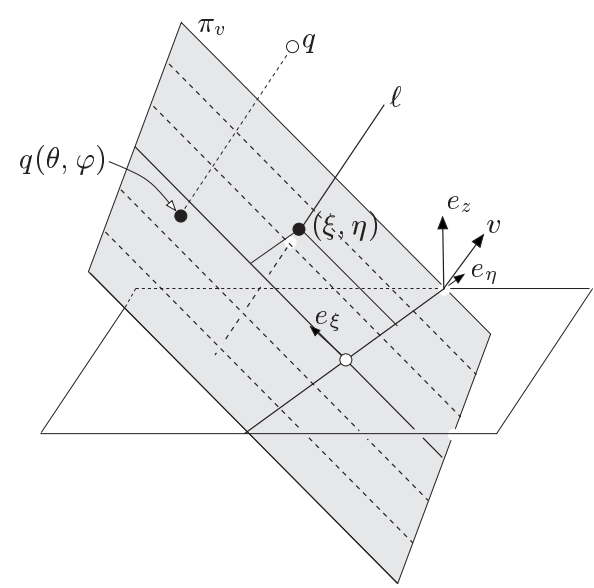

Fig. 5. Representation of lines in space; $\ell$ is represented by the quadruple $(\theta, \varphi, \xi, \eta)$.

is the horizontal orientation of the $x y$-projection of $v$. Let $\pi_{v}$ denote the plane orthogonal to $v$ and passing through the origin $o$. We define below a two-dimensional coordinate frame $(\xi, \eta)$ in $\pi_{v}$ with $o$ as its origin. The last two parameters $(\xi, \eta)$ that represent $\ell$ are the coordinates in this frame of the point $\pi_{v} \cap \ell$.

Let $e_{z}:=(0,0,1)$ be the vertical unit vector. The unit vectors $e_{\xi}, e_{\eta}$ that define the $\xi$ - and $\eta$ - axes are constructed as follows. We set

$$
e_{\eta}:=(\cos (\theta+\pi / 2), \sin (\theta+\pi / 2), 0)=(-\sin \theta, \cos \theta, 0)
$$

We observe that $e_{\eta}$ is orthogonal to both $e_{z}$ and $v$. (Note that $\theta$ is undefined when $v$ is vertical. We exclude lines with $\varphi=0$ from our analysis; with a generic choice of $u$, no vertex of $\mathcal{W}(\mathcal{B})$ will correspond to a vertical line.) We then choose $e_{\xi}:=v \times e_{\eta}$, i.e., the $\xi$-axis is the intersection of $\pi_{v}$ with the vertical plane through $o$ spanned by $v$ and $e_{z}$. See Fig. 5. For a point $q \in \mathbb{R}^{3}$ and a direction $v=(\theta, \varphi) \in \mathbb{S}^{2}$, let $q(v)=q(\theta, \varphi)$ denote the orthogonal projection of $q$ onto the plane $\pi_{v}$, whose coordinates are represented in the $(\xi, \eta)$-system. Note that the $\eta$-coordinate of $q(\theta, \varphi)$ does not depend on the value of $\varphi$. Similarly, for a ball $B \in \mathcal{B}$, we denote by $B(v)$ its orthogonal projection onto $\pi_{v}$ (which is a unit disk), represented in the $(\xi, \eta)$-system.

We choose a sufficiently small parameter $\varrho<2 \sin \alpha$, and partition the plane $\pi_{v}$ into $\xi$-vertical strips of the form

$$
\sigma_{i}^{v}=\sigma_{i}^{(\theta, \varphi)}: \varrho i \leq \eta \leq \varrho(i+1), \quad \text { for } \quad i \in \mathbb{Z} .
$$

We partition $\mathcal{D}(\mathcal{B})$ into subsets as follows. For $i \in \mathbb{Z}$, set

$$
\mathcal{D}_{i}:=\left\{w=\left(\theta_{w}, \varphi_{w}, \xi_{w}, \eta_{w}\right) \mid\left(\xi_{w}, \eta_{w}\right) \in \sigma_{i}^{\left(\theta_{w}, \varphi_{w}\right)}\right\}
$$

We bound the size of each $\mathcal{D}_{i}$ separately, as follows. Fix $i$ and define a family $\mathfrak{F}_{i}=$ $\left\{\Phi_{i 1}, \ldots, \Phi_{i n}\right\}$ of $n$ partially defined trivariate functions, one for each ball in $\mathcal{B}$, with $\theta, \varphi, \xi$ being the independent variables. A function $\Phi_{i j}$ is defined for all $(\theta, \varphi, \xi)$ such 


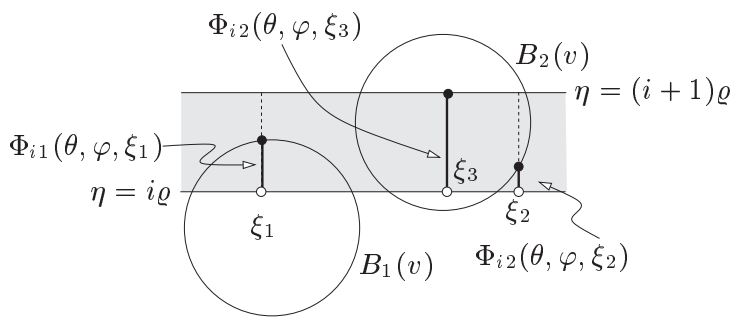

Fig. 6. Definition of the function $\Phi_{i j}(\theta, \varphi, \xi)$; all three cases are illustrated.

that one of the points $(\xi, \varrho i),(\xi, \varrho(i+1))$ in the plane $\pi_{v}$ is contained in $B_{j}(v)$, where $v:=(\theta, \varphi)$.

Specifically, the domain $\Delta_{i j}$ of $\Phi_{i j}$ is defined as follows. For a triple $(\theta, \varphi, \xi)$, let $v:=(\theta, \varphi)$ and consider the strip $\sigma_{i}^{v}$. If $K_{i j}:=B_{j}(v) \cap \sigma_{i}^{v}=\emptyset$ then $\Phi_{i j}(\theta, \varphi, \xi)$ is undefined for all $\xi$. If $K_{i j}$ is nonempty, let $I_{i j}=I_{i j}(\theta, \varphi)$ denote the set of all $\xi$ for which at least one of $(\xi, \varrho i),(\xi, \varrho(i+1))$ is in $K_{i j}$; it is easily seen that $I_{i j}$ is a nonempty interval. Then $\Phi_{i j}(\theta, \varphi, \xi)$ is defined if and only if $\xi \in I_{i j}$. Its value depends on which of the points $(\xi, \varrho i),(\xi, \varrho(i+1))$ lie in $K_{i j}$. Let $\lambda$ be the length of the intersection of $K_{i j}$ with the $\eta$-parallel line through $(\xi, \varrho i)$. If only $(\xi, \varrho i) \in K_{i j}$, then $\Phi_{i j}(\theta, \varphi, \xi)=\lambda$ (refer to $\Phi_{i 1}\left(\theta, \varphi, \xi_{1}\right)$ in Fig. 6); if only $(\xi, \varrho(i+1)) \in K$, then $\Phi_{i j}(\theta, \varphi, \xi)=\varrho-\lambda$ $\left(\Phi_{i 2}\left(\theta, \varphi, \xi_{2}\right)\right.$ in the figure); if both $(\xi, \varrho i),(\xi, \varrho(i+1)) \in K_{i j}$, then $\Phi_{i j}(\theta, \varphi, \xi)=\varrho$ $\left(\Phi_{i 2}\left(\theta, \varphi, \xi_{3}\right)\right.$ in the figure). Note that $\Phi_{i j}$ may be discontinuous at the intersection point of $\partial B_{j}(v)$ and $\eta=\varrho i$, for example, see $\Phi_{i 2}$ in Fig. 6.

Next, we partition the domain of $\Phi_{i j}$ into two subdomains $\Delta_{i j}^{+}, \Delta_{i j}^{-}$, as follows: $(\theta, \varphi, \xi) \in \Phi_{i j}$ belongs to $\Delta_{i j}^{+}$if the point $(\xi, \varrho i) \in B_{j}(v)$ and to $\Delta_{i j}^{-}$otherwise (i.e., if $(\xi, \varrho i) \notin B_{j}(v)$ and $\left.(\xi, \varrho(i+1)) \in B_{j}(v)\right)$. We denote the restriction of $\Phi_{i j}$ to $\Delta_{i j}^{+}$(resp., to $\Delta_{i j}^{-}$) as $\Phi_{i j}^{+}$(resp., as $\Phi_{i j}^{-}$). Each function $\Phi_{i j}^{+}, \Phi_{i j}^{-}$has constant description complexity, meaning that it can be defined in terms of a constant number of polynomial equalities and inequalities of constant maximum degree (using the standard re-parametrization of $\theta, \varphi$ by $\tan (\theta / 2)$ and $\tan (\varphi / 2)$, respectively).

For each $i$, set $\mathfrak{F}_{i}^{+}:=\left\{\Phi_{i j}^{+}\right\}_{j=1}^{n}$ and $\mathfrak{F}_{i}^{-}:=\left\{\Phi_{i j}^{-}\right\}_{j=1}^{n}$; only functions with nonempty domains of definition are included in these collections. Let

$$
\Xi_{i}:=\left\{(\theta, \varphi, \xi, \eta) \in \mathbb{S}^{2} \times \mathbb{R}^{2} \mid \max _{j} \Phi_{i j}^{+}(\theta, \varphi, \xi) \leq \eta \leq \min _{j} \Phi_{i j}^{-}(\theta, \varphi, \xi)\right\}
$$

$\Xi_{i}$ is a "sandwich region," i.e., the region lying between the upper envelope of $\mathfrak{F}_{i}^{+}$and the lower envelope of $\mathfrak{F}_{i}^{-}$. Set $n_{i}=\left|\mathfrak{F}_{i}^{-}\right|+\left|\mathfrak{F}_{i}^{+}\right|$. We call a vertex of $\Xi_{i}$ rainbow if the four function graphs incident upon the vertex correspond to balls from different families $\mathcal{B}_{j}$.

Lemma 4.1 (Sandwich Lemma). Each rainbow vertex of $\mathcal{D}_{i}$ corresponds to a distinct rainbow vertex of $\Xi_{i}$.

Proof. This is a consequence of the definition of deep vertices. Let $w=\left(\theta_{w}, \varphi_{w}, \xi_{w}, \eta_{w}\right)$ be a deep vertex; set $v_{w}=\left(\theta_{w}, \varphi_{w}\right)$. The projection of each of the four balls $B \in \mathcal{B}_{w}$ is a 
unit disk in $\pi_{v_{w}}$ and the line $\tau_{w}$ intersects $\pi_{v_{w}}$ at a point $w^{*}$ lying on the boundaries of all four disks. Since $w$ is deep, the $\eta$-parallel line through $w^{*}$, whose direction is orthogonal to both $v_{w}$ and the $z$-direction, intersects each of these disks in a chord of length at least $2 \sin \alpha>\varrho$; see Fig. 2. This implies that each of the four corresponding functions $\Phi_{i j}$ (where $i$ is the index of the strip that contains $w^{*}$ is defined at $\left(\theta_{w}, \varphi_{w}, \xi_{w}\right)$, and they all have the same value there. Moreover, since no other ball projects to a disk that contains $w^{*}$, it follows that the intersection of the graphs of the four $\Phi_{i j}$ 's is indeed a (rainbow) vertex of $\Xi_{i}$.

By the result of Koltun and Sharir [13], the number of vertices of $\Xi_{i}$ is $O\left(n_{i}^{3+\varepsilon}\right)$, for any $\varepsilon>0$. Summing over all strips $\sigma_{i}^{v}$, the overall number of deep vertices under consideration is $\sum_{i \in \mathbb{Z}} O\left(n_{i}^{3+\varepsilon}\right)$, for any $\varepsilon>0$.

Unfortunately, this bound is too weak-in fact, it can be arbitrarily large, because, if the center of a ball $B_{j}$ lies at distance $r$ from $o, B_{j}$ contributes a function $\Phi_{i j}$ with nonempty domain of definition to $\Theta(r / \varrho)$ strips, so if the balls lie arbitrarily far from the origin, the number of indices $i$ with $n_{i} \approx n$ may be arbitrarily large. We handle this problem below by counting only the number of rainbow vertices, exploiting the separation property of $\mathcal{B}$, and refining the bound of [13].

\subsection{Exploiting Separation}

Let $J_{i j}$ be the $\theta$-projection of $\Delta_{i j}$, and let $\chi_{i}$ be the number of rainbow triples $B_{1}, B_{2}, B_{3} \in$ $\mathcal{B}$ so that $J_{i 1} \cap J_{i 2} \cap J_{i 3} \neq \emptyset$, i.e., there exist $\theta_{0}, \varphi_{j}, \xi_{j}$, for $1 \leq j \leq 3$, with $\left(\theta_{0}, \varphi_{j}, \xi_{j}\right) \in$ $\Delta_{i j}$ for each $j$. Our refinement, obtained in the following section (Corollary 5.4), shows that the number of rainbow vertices in $\Xi_{i}$ is $O\left(n^{\varepsilon} \chi_{i}\right)$. We will show (see Lemma 4.4) that $\sum_{i} \chi_{i}=O\left(n^{3}\right)$, which will then imply that the total number of deep rainbow vertices in $\mathcal{F}(\mathcal{B})$ is $O\left(n^{3+\varepsilon}\right)$, and thus complete the proof of Theorem 1.1.

The following is a key technical lemma that encapsulates the significance of separation for our analysis.

Lemma 4.2 (Sparseness Lemma). There exists a constant $c=c(\varrho)$ with the following property. For any pair $\left\{B_{1}, B_{2}\right\}$ of distinct unit balls that are well-separated with respect to the origin, there are at most $c$ integers $k$ with the property that $J_{k 1} \cap J_{k 2} \neq \emptyset$, i.e., the $\theta$-projections of $\Delta_{k 1}$ and $\Delta_{k 2}$ overlap.

Proof. Let $\mathcal{I}$ denote the set of integers $k$ for which $J_{k 1} \cap J_{k 2} \neq \emptyset$. Let $k \in \mathcal{I}$, and let $\theta_{0} \in J_{k 1} \cap J_{k 2}$. Recall that the $\eta$-direction in $\pi_{v}$ depends only on $\theta$ and not on $\varphi$. Consequently, if we keep $\theta_{0}$ fixed and vary $\varphi$, the plane $\pi_{v}$ rotates about the fixed horizontal $\eta$-axis. For any fixed point $q \in \mathbb{R}^{3}$, its projection onto $\pi_{v}$ traces a vertical circular arc in $\mathbb{R}^{3}$, which appears within the rotating plane $\pi_{v}$ as a line segment orthogonal to the $\eta$-axis. Note also that the $k$ th strip $\sigma_{k}^{v}$ rotates with $\pi_{v}$ about the fixed $\eta$-axis, but its representation in the moving $(\xi, \eta)$-system does not change.

Let $c_{1}, c_{2}$ be the respective centers of $B_{1}, B_{2}$. Since $\theta_{0} \in J_{k 1} \cap J_{k 2}$, the two (parallel) segments traced within $\pi_{v}$ by the projections of $c_{1}$ and $c_{2}$ lie at $\eta$-distance at most one 
from the (fixed) strip $\sigma_{k}^{v}$. In particular, when $\pi_{v}$ becomes horizontal, both $x y$-projections $q_{1}=c_{1}^{*}, q_{2}=c_{2}^{*}$ of these centers lie in the $x y$-plane, each at $\eta$-distance at most one from $\sigma_{k}^{\left(\theta_{0}, 0\right)}$ and thus at $\eta$-distance at most $2+\varrho$ from each other. Technically, we need to regard the horizontal position of $\pi_{v}$ as a limit position, obtained as $\varphi \rightarrow 0$, because the $(\xi, \eta)$-system is undefined when $\varphi=0$. We interpret $\sigma_{k}^{\left(\theta_{0}, 0\right)}$ simply as strips orthogonal to the line at orientation $\left(-\sin \theta_{0}, \cos \theta_{0}, 0\right)$. Our goal is thus to bound the number of integers $k$ for which there exists $\theta$ such that both points $q_{1}$ and $q_{2}$ lie in the extended strip $\hat{\sigma}_{k}^{(\theta, 0)}$ of width $2+\varrho$, obtained by expanding $\sigma_{k}^{(\theta, 0)}$ by distance one in the positive and negative $\eta$-directions. Note that $\hat{\sigma}_{k}^{(\theta, 0)}$ rotates about the origin as $\theta$ varies. We distinguish between two cases:

Case (i): $\left|q_{1} q_{2}\right| \leq 2+\varrho$. Since $B_{1}, B_{2}$ are well-separated, $\left|o q_{1}\right| \leq\left|q_{1} q_{2}\right| \leq 2+\varrho$. Hence any strip $\hat{\sigma}_{k}^{(\theta, 0)}$ that contains $q_{1}$ must lie at distance at most $2+\varrho$ from the origin, and the number of such strips is at most $2\lceil(3+\varrho) / \varrho\rceil$. This clearly also serves as an upper bound on the number of extended strips that contain (for the same $\theta$ ) both $q_{1}$ and $q_{2}$.

Case (ii): $\left|q_{1} q_{2}\right|>2+\varrho$. Since the situation is symmetric with respect to rotation about the origin, we may assume, without loss of generality, that $q_{1}$ and $q_{2}$ have the same $y$-coordinate $h$. Since $B_{1}$ and $B_{2}$ are well-separated, $q_{1} q_{2}$ is the longest edge in the triangle $o q_{1} q_{2}$, which implies that $q_{1}$ and $q_{2}$ lie in different quadrants, and that $h \leq\left|q_{1} q_{2}\right|$. See Fig. 7. A point $x \in \hat{\sigma}_{k}^{(\theta, 0)}$ must satisfy

$$
k \varrho-1 \leq x \cdot e_{\eta} \leq(k+1) \varrho+1 .
$$

In particular, as the extended strip $\hat{\sigma}_{k}^{(\theta, 0)}$ rotates about the origin, it contains both $q_{1}$ and $q_{2}$ when the corresponding $e_{\eta}$ satisfies

$$
\begin{aligned}
& k \varrho-1 \leq q_{1} \cdot e_{\eta} \leq(k+1) \varrho+1 \quad \text { and } \\
& k \varrho-1 \leq q_{2} \cdot e_{\eta} \leq(k+1) \varrho+1 .
\end{aligned}
$$

Subtracting the two inequalities, we obtain

$$
\left|q_{1} q_{2} \cdot e_{\eta}\right| \leq(k+1) \varrho+1-(k \varrho-1)=2+\varrho .
$$

On the other hand,

$$
\left|q_{1} q_{2} \cdot e_{\eta}\right|=\left|q_{1} q_{2} \cdot(-\sin \theta, \cos \theta, 0)\right|=\left|q_{1} q_{2}\right||\sin \theta| .
$$

Therefore

$$
|\sin \theta| \leq \frac{2+\varrho}{\left|q_{1} q_{2}\right|}
$$

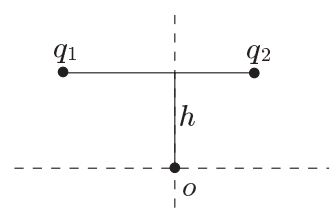

Fig. 7. The triangle $o q_{1} q_{2}$. 
In addition, since both $q_{1}, q_{2} \in \hat{\sigma}_{k}^{(\theta, 0)}$, so is the point $(0, h, 0)$, which must therefore also satisfy (3), namely,

$$
k \varrho-1 \leq h \cos \theta \leq(k+1) \varrho+1 .
$$

Let $\theta_{0} \in(0, \pi / 2)$ satisfy $\sin \theta_{0}=(2+\varrho) /\left|q_{1} q_{2}\right|$. The range of $\theta$ where (5) holds is thus contained in the union of the two angular intervals $\left(-\theta_{0}, \theta_{0}\right)$ and $\left(\pi-\theta_{0}, \pi+\theta_{0}\right)$. Any $k$ that satisfies (4.2), and thus also (6), must therefore be in one of the two ranges

$$
\left[\min _{\theta \in\left(-\theta_{0}, \theta_{0}\right)} \frac{h \cos \theta-1}{\varrho}-1, \max _{\theta \in\left(-\theta_{0}, \theta_{0}\right)} \frac{h \cos \theta+1}{\varrho}\right]
$$

and

$$
\left[\min _{\theta \in\left(\pi-\theta_{0}, \pi+\theta_{0}\right)} \frac{h \cos \theta-1}{\varrho}-1, \max _{\theta \in\left(\pi-\theta_{0}, \pi+\theta_{0}\right)} \frac{h \cos \theta+1}{\varrho}\right] .
$$

The number of integers $k$ in the former range is at most

$$
\begin{aligned}
& \left\lceil\frac{h}{\varrho}\left(\max _{\theta \in\left(-\theta_{0}, \theta_{0}\right)} \cos \theta-\min _{\theta \in\left(-\theta_{0}, \theta_{0}\right)} \cos \theta\right)+\frac{2}{\varrho}+1\right\rceil \\
& =\left\lceil\frac{h\left(1-\cos \theta_{0}\right)}{\varrho}+\frac{2}{\varrho}+1\right\rceil<\left\lceil\frac{h \sin \theta_{0}}{\varrho}+\frac{2}{\varrho}+1\right\rceil \leq\left\lceil\frac{4}{\varrho}+2\right\rceil,
\end{aligned}
$$

where we have used the facts that $h \leq\left|q_{1} q_{2}\right|$ and that $\left|q_{1} q_{2}\right| \sin \theta_{0}=2+\varrho$. The analysis for the second angular interval yields the same estimate, for a total of no more than $2(4 / \varrho+3)$ values of $k$. This completes the proof of the lemma.

Corollary 4.3. There exists a constant $c=c(\varrho)$ with the following property. For any triple $\left\{B_{1}, B_{2}, B_{3}\right\}$ of distinct unit balls such that $B_{1}$ and $B_{2}$ are well-separated with respect to the origin, there are at most $c$ integers $k$ with the property that $J_{k 1} \cap J_{k 2} \cap$ $J_{k 3} \neq \emptyset$.

Lemma 4.4. $\sum_{i} \chi_{i}=O\left(n^{3}\right)$.

Proof.

$$
\begin{aligned}
\sum_{i} \chi_{i} & =\sum_{i} \mid\left\{(a, b, c) \mid a<b<c \text { and } J_{i a} \cap J_{i b} \cap J_{i c} \neq \emptyset\right\} \mid \\
& =\sum_{a<b<c}\left|\left\{i \mid J_{i a} \cap J_{i b} \cap J_{i c} \neq \emptyset\right\}\right| \\
& \leq\left(\begin{array}{c}
n \\
3
\end{array}\right) c(\varrho)=O\left(n^{3}\right) .
\end{aligned}
$$

As already mentioned, in Corollary 5.4 in the following section we prove that $\left|\Xi_{i}\right|=$ $O\left(n^{\varepsilon} \chi_{i}\right)$, making crucial use of the separation property-see below for details. This implies the following result, which serves as the missing link in the proof of Theorem 1.1, as presented in Sections 2 and 3. 
Lemma 4.5. Let $\mathcal{B}_{1}, \mathcal{B}_{2}, \mathcal{B}_{3}, \mathcal{B}_{4}$ be four sets of unit balls in $\mathbb{R}^{3}$ of total size $n$ which are doubly well-separated with respect to the origin. Then the number of deep rainbow vertices in $\mathcal{F}\left(\mathcal{B}_{1} \cup \mathcal{B}_{2} \cup \mathcal{B}_{3} \cup \mathcal{B}_{4}\right)$ is $O\left(n^{3+\varepsilon}\right)$, for any $\varepsilon>0$.

\section{Functions with Sparse Domains}

This section provides refined upper bounds on the complexity of the "sandwich" region of two collections of trivariate partially defined functions. We believe that this refinement is of independent interest and expect it to be useful for other applications as well. Let $\mathcal{F}=$ $\left\{f_{1}, \ldots, f_{n}\right\}$ be a family of partially defined trivariate functions of constant description complexity (as defined in the preceding section) and in general position, which is the disjoint union $\mathcal{F}_{1} \cup \mathcal{F}_{2} \cup \mathcal{F}_{3} \cup \mathcal{F}_{4}$ of four subfamilies. In what follows we do not distinguish between a function and its graph. We call a subset $\mathcal{R} \subseteq \mathcal{F}$ of size at most four rainbow if each function in $\mathcal{R}$ belongs to a different set $\mathcal{F}_{j}$. A vertex in the arrangement $\mathcal{A}(\mathcal{F})$ of (the graphs of) the functions in $\mathcal{F}$ is called rainbow if the subset of four functions whose graphs are incident upon $v$ is rainbow. Let $\mathcal{F}^{+}$and $\mathcal{F}^{-}$be another partition of $\mathcal{F}$ into two subsets, and let $\Xi\left(\mathcal{F}^{+}, \mathcal{F}^{-}\right)$denote the sandwich region lying between the upper envelope of $\mathcal{F}^{+}$and the lower envelope of $\mathcal{F}^{-}$, i.e.,

$$
\Xi\left(\mathcal{F}^{+}, \mathcal{F}^{-}\right):=\left\{(x, y, z, w) \in \mathbb{R}^{4} \mid \max _{f \in \mathcal{F}^{+}} f(x, y, z) \leq w \leq \min _{f \in \mathcal{F}^{-}} f(x, y, z)\right\} .
$$

Let $\Pi$ be the set of rainbow vertices of $\Xi\left(\mathcal{F}^{+}, \mathcal{F}^{-}\right)$. We wish to bound the size of $\Pi$. As already noted, the recent result of [13] implies that the size of $\Pi$ is $O\left(n^{3+\varepsilon}\right)$, for any $\varepsilon>0$. The goal of this section is to refine this bound in terms of a parameter that counts overlaps between the domains of the functions in $\mathcal{F}$, in a manner already alluded to in the previous section and defined more precisely below. We begin with the following simple but weak bound, which is central in the derivation of our main result.

Lemma 5.1. If $\left|\mathcal{F}_{1}\right| \geq\left|\mathcal{F}_{2}\right| \geq\left|\mathcal{F}_{3}\right| \geq\left|\mathcal{F}_{4}\right|$, then $|\Pi|=O\left(\left|\mathcal{F}_{1}\right|\left|\mathcal{F}_{2}\right|\left|\mathcal{F}_{3}\right|^{1+\varepsilon}\right)$, for any $\varepsilon>0$.

Proof. Put $n_{i}=\left|\mathcal{F}_{i}\right|$, for $i=1, \ldots, 4$. Partition $\mathcal{F}_{1}$ into $t:=\left\lceil n_{1} / n_{3}\right\rceil$ pairwisedisjoint subsets $X_{1}, \ldots, X_{t}$, each of size at most $n_{3}$, and partition similarly $\mathcal{F}_{2}$ into $s:=\left\lceil n_{2} / n_{3}\right\rceil$ pairwise-disjoint subsets $Y_{1}, \ldots, Y_{s}$, each also of size at most $n_{3}$. Let $\mathcal{F}_{i j}^{+}:=\mathcal{F}^{+} \cap\left(X_{i} \cup Y_{j} \cup \mathcal{F}_{3} \cup \mathcal{F}_{4}\right)$ and $\mathcal{F}_{i j}^{-}:=\mathcal{F}^{-} \cap\left(X_{i} \cup Y_{j} \cup \mathcal{F}_{3} \cup \mathcal{F}_{4}\right)$, for $i=1, \ldots, t$ and $j=1, \ldots, s$. Then any vertex of $\Pi$ is a vertex of $\Xi\left(\mathcal{F}_{i j}^{+}, \mathcal{F}_{i j}^{-}\right)$for some $i$ and $j$. Since $\left|\mathcal{F}_{i j}^{+} \cup \mathcal{F}_{i j}^{-}\right| \leq 4 n_{3}$, the aforementioned bound of [13] implies that $\Xi\left(\mathcal{F}_{i j}^{+}, \mathcal{F}_{i j}^{-}\right)$has $O\left(n_{3}^{3+\varepsilon}\right)$ vertices, for any $\varepsilon>0$. Hence, the overall number of vertices in $\Pi$ is at most $t s \cdot O\left(n_{3}^{3+\varepsilon}\right)=O\left(n_{1} n_{2} n_{3}^{1+\varepsilon}\right)$, for any $\varepsilon>0$, as asserted.

For each $i=1, \ldots, n$, let $\Delta_{i}$ be the domain of $f_{i}$, and let $J_{i}$ denote the $z$-projection (i.e., the orthogonal projection onto the $z$-axis) of $\Delta_{i}$. Without loss of generality, we assume that $J_{i}$ is a connected interval; otherwise we decompose $f_{i}$ into $O(1)$ further partially defined functions so that each of them satisfies this property. Let $E$ denote the 
set of endpoints of intervals $J_{i}$, for $1 \leq i \leq n$. For the sake of simplicity, we assume that all the endpoints are distinct, but the analysis can be easily adapted to handle degenerate cases as well.

Let $\chi$ be the number of rainbow triples $\left(f_{i}, f_{l}, f_{k}\right)$ so that $J_{i} \cap J_{l} \cap J_{k} \neq \emptyset$. This is our (rather weak) measure of the amount of overlap between the domains of the given functions. Our goal is to bound $|\Pi|$ in terms of $\chi$.

Let $\Sigma \subseteq E \times \mathcal{F} \times \mathcal{F}$ be the set of triples $\left(p, f_{l}, f_{k}\right)$ so that $p \in J_{l} \cap J_{k}$, and the function $f_{i}$ for which $p$ is an endpoint of $J_{i}$ forms a rainbow triple with $f_{l}$ and $f_{k}$. Set $\sigma_{0}:=|\Sigma|=2 \chi$.

We use a recursive scheme to bound $|\Pi|$. At each recursive step, we have an open $z$-interval $Z$. Let $\mathcal{F}_{Z}:=\left\{f_{i} \in \mathcal{F} \mid J_{i} \cap Z \neq \emptyset\right\}$. A function $f_{i} \in \mathcal{F}_{Z}$ is called short in $Z$ if at least one of the endpoints of $J_{i}$ lies in $Z$; otherwise, $f_{i}$ is long. Let $\mathcal{L}_{Z}$ (resp., $\mathcal{S}_{Z}$ ) denote the set of long (resp., short) functions in $Z$. Let $\Pi_{Z} \subseteq \Pi$ denote the subset of vertices $w \in \Pi$ whose $z$-coordinates lie in $Z$ and are such that at least two of the four functions whose graphs are incident upon $w$ are in $\mathcal{S}_{Z}$. Initially, we set $Z=(-\infty, \infty)$, $S_{Z}=\mathcal{F}$, and $\mathcal{L}_{Z}=\emptyset$. Therefore $\Pi_{Z}=\Pi$ for $Z=(-\infty, \infty)$. It thus suffices to bound the size of $\Pi_{Z}$.

For a $z$-interval $Z$, let $E_{Z}:=E \cap Z, \Sigma_{Z}:=\Sigma \cap\left(E_{Z} \times \mathcal{F}_{Z} \times \mathcal{F}_{Z}\right)$, and $\sigma_{Z}:=\left|\Sigma_{Z}\right|$. Let $\Psi(m, \sigma):=\max \left|\Pi_{Z}\right|$, where the maximum is taken over all $z$-intervals $Z$ with $\left|E_{Z}\right| \leq m$ and $\left|\Sigma_{Z}\right| \leq \sigma$. We derive a recurrence for $\Psi(m, \sigma)$ by partitioning an interval $Z$ into two subintervals $Z_{1}$ and $Z_{2}$, each containing roughly the same number of endpoints of $E_{Z}$. The overhead term in the recurrence accounts for the number of vertices in $\Pi_{Z} \backslash\left(\Pi_{Z_{1}} \cup \Pi_{Z_{2}}\right)$, i.e., the vertices $w$ such that at least two of the four functions whose graphs are incident upon $w$ are short in $Z$ but only at most one of these functions is short in $Z_{1}$ or $Z_{2}$, whichever contains the $z$-coordinate of $w$. We prove that the overhead term is roughly $\sigma_{Z}$ and observe that $\sigma_{Z} \leq \sigma_{Z_{1}}+\sigma_{Z_{2}}$. Putting these facts together we obtain a bound on $|\Pi|$ in terms of $\chi$. We now provide a detailed analysis that follows this general outline.

Let $Z$ be a $z$-interval as above, with $\left|E_{Z}\right| \leq m$ and $\left|\Sigma_{Z}\right| \leq \sigma$. If $\mathcal{S}_{Z}=\emptyset$, then $\left|\Pi_{Z}\right|=0$. Otherwise we partition $Z$ into two intervals $Z_{1}, Z_{2}$ so that each subinterval contains half of the endpoints in $E_{Z}$. For $i=1,2$, set $\mathcal{F}_{i}:=\mathcal{F}_{Z_{i}}, \mathcal{L}_{i}:=\mathcal{L}_{Z_{i}}, \mathcal{S}_{i}:=\mathcal{S}_{Z_{i}}$, $E_{i}:=E_{Z_{i}}, \Sigma_{i}:=\Sigma_{Z_{i}}$, and $\sigma_{i}:=\left|\Sigma_{i}\right|$. We also denote by $\mathcal{N}_{i} \subseteq \mathcal{L}_{i}$ the set of functions that are long in $Z_{i}$ but short in $Z$. Note that $\mathcal{S}_{i} \cup \mathcal{N}_{i}$ is the set of all functions $f_{l} \in \mathcal{S}_{Z}$ such that $J_{l} \cap Z_{i} \neq \emptyset$. Let $w \in \Pi_{Z}$ be a vertex whose $z$-coordinate lies in $Z_{1}$, say. If $w$ is incident upon at least two functions in $\mathcal{S}_{1}$, then $w \in \Pi_{Z_{1}}$ and is counted recursively in the subproblem associated with $Z_{1}$. Otherwise, since $w$ is assumed to be incident upon at least two short functions in $\mathcal{S}_{Z}, w$ is incident upon at least one function in $\mathcal{N}_{1}$, at most one function in $\mathcal{S}_{1}$, and at most two functions in $\mathcal{L}_{1} \backslash \mathcal{N}_{1}$. In fact, enumerating all possible cases, $w$ must be incident upon one function in $\mathcal{N}_{1}$, another function in $\mathcal{S}_{1} \cup \mathcal{N}_{1}$, and two functions in $\mathcal{L}_{1}$ (any other combination would pass $w$ to the recursive subproblem at $Z_{1}$ ). Let $\Pi_{1}^{\prime} \subseteq \Pi_{Z}$ be the set of such vertices. We define $\Pi_{2}^{\prime}$ for the other subinterval $Z_{2}$ in a fully analogous manner. We thus obtain the following recurrence for $\Psi(m, \sigma)$ :

$$
\Psi(m, \sigma) \leq \begin{cases}\Psi\left(m / 2, \sigma_{1}\right)+\Psi\left(m / 2, \sigma_{2}\right)+\left|\Pi_{1}^{\prime}\right|+\left|\Pi_{2}^{\prime}\right|, & \sigma>0, \\ 0, & \sigma=0 .\end{cases}
$$


Since $E_{1} \cap E_{2}=\emptyset, \sigma_{1}+\sigma_{2} \leq \sigma$. We now establish the following bound on $\left|\Pi_{1}^{\prime}\right|$ and $\left|\Pi_{2}^{\prime}\right|$.

Lemma 5.2. For $i=1,2,\left|\Pi_{i}^{\prime}\right|=O\left(\sigma_{Z} n^{\varepsilon}\right)$, for any $\varepsilon>0$.

Proof. $\quad$ Let $w \in \Pi_{1}^{\prime}$ be a vertex incident upon $f_{1} \in \mathcal{F}_{1}, f_{2} \in \mathcal{F}_{2}, f_{3} \in \mathcal{F}_{3}$, and $f_{4} \in \mathcal{F}_{4}$. By definition, we may assume that, up to a permutation, $f_{1}, f_{2} \in \mathcal{L}_{1}, f_{3} \in \mathcal{N}_{1}$, and $f_{4} \in \mathcal{S}_{1} \cup \mathcal{N}_{1}$. Set

$$
\begin{array}{ll}
X_{1}:=\mathcal{F}_{1} \cap \mathcal{L}_{1}, & X_{3}:=\mathcal{F}_{3} \cap \mathcal{N}_{1}, \\
X_{2}:=\mathcal{F}_{2} \cap \mathcal{L}_{1}, & X_{4}:=\mathcal{F}_{4} \cap\left(\mathcal{S}_{1} \cup \mathcal{N}_{1}\right) .
\end{array}
$$

By Lemma 5.1, the number of those rainbow vertices $w \in \Xi\left(\mathcal{F}^{+}, \mathcal{F}^{-}\right)$that are incident upon one function in each of $X_{1}, X_{2}, X_{3}, X_{4}$ is $O\left(n^{\varepsilon}\right)$ times the product of the three largest sizes of the sets $X_{i}$, for any $\varepsilon>0$. Hence this number is definitely upper bounded by $O\left(n^{\varepsilon} \sum_{i<j<k}\left|X_{i}\right|\left|X_{j}\right|\left|X_{k}\right|\right)$.

Let $f_{i} \in X_{i}, f_{j} \in X_{j}, f_{k} \in X_{k}$. Since at least two of these functions are long in $Z_{1}$, it follows that $J:=J_{i} \cap J_{j} \cap J_{k} \neq \emptyset$. Moreover, since at least one of these functions is short in $Z, J$ must have an endpoint $p$ in $Z$, which is an endpoint of, say, $J_{k}$. We then charge $\left(f_{i}, f_{j}, f_{k}\right)$ to the triple $\left(p, f_{i}, f_{j}\right)$, which is counted in $\Sigma_{Z}$. It is easily seen that each triple in $\Sigma_{Z}$ is charged at most $O(1)$ times in this manner. Hence we have

$$
\sum_{i<j<k}\left|X_{i}\right|\left|X_{j}\right|\left|X_{k}\right|=O\left(\sigma_{Z}\right) .
$$

Repeating this argument for all other $O(1)$ types of vertices in $\Pi_{1}^{\prime}$ (obtained by permuting the sets $\mathcal{F}_{1}, \ldots, \mathcal{F}_{4}$, and/or the short/long classification of the four functions incident upon the vertex), we can conclude that $\left|\Pi_{1}^{\prime}\right|=O\left(n^{\varepsilon} \sigma_{Z}\right)$, for any $\varepsilon>0$. Using a fully symmetric argument, it follows that $\left|\Pi_{2}^{\prime}\right|=O\left(n^{\varepsilon} \sigma_{Z}\right)$, for any $\varepsilon>0$. This completes the proof of the lemma.

Plugging the bounds on $\left|\Pi_{1}^{\prime}\right|$ and $\left|\Pi_{2}^{\prime}\right|$ into the recurrence, we obtain that

$$
\Psi(m, \sigma) \leq \begin{cases}\Psi\left(m / 2, \sigma_{1}\right)+\Psi\left(m / 2, \sigma_{2}\right)+O\left(n^{\varepsilon} \sigma\right), & \sigma>0, \\ 0, & \sigma=0,\end{cases}
$$

where $\sigma=\sigma_{1}+\sigma_{2}$. The solution of this recurrence is

$$
\Psi(m, \sigma)=O\left(n^{\varepsilon} \sigma \log m\right) .
$$

Initially, $Z=(-\infty,+\infty), m=|E|=2 n$, and $\sigma_{0}=|\Sigma|=2 \chi$. Therefore

$$
|\Pi| \leq \Psi(2 n, 2 \chi)=O\left(n^{\varepsilon} \chi \log n\right)=O\left(\chi n^{\varepsilon^{\prime}}\right),
$$

for any $\varepsilon^{\prime}>\varepsilon$. Putting everything together, we obtain the following result.

Theorem 5.3. Let $\mathcal{F}$ be a family of $n$ partially defined trivariate functions of constant description complexity, and let $\left\{\mathcal{F}_{1}, \mathcal{F}_{2}, \mathcal{F}_{3}, \mathcal{F}_{4}\right\}$ be a partition of $\mathcal{F}$. Let $\chi$ denote the 
number of rainbow triples of functions in $\mathcal{F}$ such that the z-projections of their domains have a common intersection. Then, for any partition $\mathcal{F}^{+}, \mathcal{F}^{-}$of $\mathcal{F}$ into two subsets, the number of rainbow vertices in the sandwich region $\Xi\left(\mathcal{F}^{+}, \mathcal{F}^{-}\right)$between the upper envelope of $\mathcal{F}^{+}$and the lower envelope of $\mathcal{F}^{-}$is $O\left(\chi n^{\varepsilon}\right)$, for any $\varepsilon>0$.

Let $\mathfrak{F}_{i}^{+}, \mathfrak{F}_{i}^{-}$be the sets of partial trivariate functions defined for the $i$ th strip in the previous section. Letting the $\theta$-axis play the role of the $z$-axis, we obtain the following last ingredient in the proof of Theorem 1.1.

Corollary 5.4. For any $i \in \mathbb{Z}$, let $\mathfrak{F}_{i}^{+}, \mathfrak{F}_{i}^{-}$, and $\chi_{i}$ be as defined in the previous section. The number of (deep) rainbow vertices in $\Xi\left(\mathfrak{F}_{i}^{+}, \mathfrak{F}_{i}^{-}\right)$is $O\left(n^{\varepsilon} \chi_{i}\right)$, for any $\varepsilon>0$.

It is easy to extend Theorem 5.3 to the following "uncolored" version which is not needed for our analysis, but may be more appropriate for future applications.

Theorem 5.5. Let $\mathcal{F}$ be a set of $n$ partially defined trivariate functions of constant description complexity. Let $\mathcal{F}^{+}, \mathcal{F}^{-}$be a partition of $\mathcal{F}$ into two subsets. Let $\chi$ be the number of triples of functions in $\mathcal{F}$ such that the z-projections of their domains have a common intersection point. Then the number of vertices in $\Xi\left(\mathcal{F}^{+}, \mathcal{F}^{-}\right)$is $O\left(\chi n^{\varepsilon}\right)$.

Proof. Partition $\mathcal{F}$ into four subfamilies $\mathcal{F}_{1}, \ldots, \mathcal{F}_{4}$ at random, assigning each function to each color class independently with probability $1 / 4$, argue that the expected number of rainbow vertices of $\Xi\left(\mathcal{F}^{+}, \mathcal{F}^{-}\right)$is at least a constant fraction of the size of $\Xi\left(\mathcal{F}^{+}, \mathcal{F}^{-}\right)$, and invoke Theorem 5.3. The number $\chi$ of course also bounds the number of rainbow triples with overlapping domains.

Remark 5.6. An interesting open problem is whether the theorems also hold when $\chi$ counts the potentially smaller number of triples of functions whose actual domains (as opposed to their $z$-projections) have a nonempty intersection. In fact, even the analogous problem for bivariate functions is still open: Can one express the complexity of the lower envelope of $n$ partial bivariate functions of constant description complexity (or of the sandwich region between two envelopes) in terms of the number $\chi$ of pairs of functions whose domains have nonempty intersection? This can be done if $\chi$ counts the number of pairs of functions for which the $y$-projections of their domains overlap, using a considerably simpler variant of the preceding analysis.

\section{Open Problems}

The paper raises many interesting and challenging open problems, some of which have already been noted above:

(i) Devise an algorithm that constructs $\mathcal{F}(\mathcal{B})$ in near-cubic time. This would have algorithmic implications for some ray shooting and motion planning problems. 
(ii) We note that our bound is not known to be nearly worst-case tight. Although the best (and trivial) construction of which we are aware yields a lower bound of $\Omega\left(n^{2}\right)$, we conjecture that the lower bound is $\Omega\left(n^{3}\right)$.

(iii) It would be interesting to extend the analysis to families $\mathcal{B}$ of other classes of objects. The simplest such extensions would be to families of balls of arbitrary radii. Another extension would be to families of "fat" objects; see for example [6]. Our analysis extends to the case where the balls in $\mathcal{B}$ are "nearly congruent," in the sense that the ratio between the largest and the smallest radii is bounded by some fixed constant, but we do not know how to extend it to the case where there is no such restriction.

\section{Acknowledgments}

The authors express their appreciation of the city of Heidelberg, whose magic ambiance was the fertile soil in which, in March 2003, the initial seeds of this work were planted and began to grow.

\section{References}

1. P. K. Agarwal and M. Sharir, Pipes, cigars, and kreplach: the union of Minkowski sums in three dimensions, Discrete Comput. Geom. 24 (2000), 645-685.

2. B. Aronov, A. Efrat, V. Koltun, and M. Sharir, On the union of $\kappa$-round objects in three and four dimensions, in Proc. 20th Annu. Sympos. Comput. Geom., 2004, pp. 383-390.

3. B. Aronov and M. Sharir, On translational motion planning of a convex polyhedron in 3-space, SIAM J. Comput. 26 (1997), 1785-1803.

4. B. Aronov, M. Sharir, and B. Tagansky, The union of convex polyhedra in three dimensions, SIAM J. Comput. 26 (1997), 1670-1688.

5. M. de Berg, H. Everett, and L. J. Guibas, The union of moving polygonal pseudodiscs—combinatorial bounds and applications, Comput. Geom. Theory Appl. 11 (1998), 69-82.

6. M. de Berg, M. J. Katz, A. F. van der Stappen, and J. Vleugels, Realistic input models for geometric algorithms, Algorithmica 34 (2002), 81-97.

7. J.-D. Boissonnat, M. Sharir, B. Tagansky, and M. Yvinec, Voronoi diagrams in higher dimensions under certain polyhedral distance functions, Discrete Comput. Geom. 19 (1998), 485-519.

8. B. Chazelle, H. Edelsbrunner, L. Guibas, M. Sharir, and J. Stolfi, Lines in space: combinatorics and algorithms, Algorithmica 15 (1996), 428-447.

9. O. Devillers, V. Dujmović, H. Everett, X. Goaoc, S. Lazard, H.-S. Na, and S. Petitjean, The expected number of 3D visibility events is linear, SIAM J. Comput. 32 (2003), 1586-1620.

10. F. Durand, G. Drettakis, and C. Puech, The 3D visibility complex, ACM Trans. Graphics, 21 (2002), 176-206.

11. A. Efrat, The complexity of the union of $(\alpha, \beta)$-covered objects, in Proc. 15th Annu. ACM Sympos. Comput. Geom., 1999, pp. 134-142.

12. A. Efrat and M. Sharir, The complexity of the union of fat objects in the plane, Discrete Comput. Geom. 23 (2000), 171-189.

13. V. Koltun and M. Sharir, The partition technique for overlays of envelopes, SIAM J. Comput. 32 (2003), 841-863.

14. J. Pach, I. Safruti, and M. Sharir, The union of congruent cubes in three dimensions, Discrete Comput. Geom. 30 (2003), 133-160.

15. M. Pellegrini, On lines missing polyhedral sets in 3-space, Discrete Comput. Geom. 12 (1994), 203-221. 
16. M. Pocchiola and G. Vegter, The visibility complex, Internat. J. Comput. Geom. Appl. 6 (1996), 279-308.

17. M. Sharir, Algorithmic motion planning, in Handbook of Discrete and Computational Geometry, 2nd edn. (J. Goodman and J. O’Rourke, eds.), pp. 1037-1064, CRC Press, Boca Raton, FL, 2004.

18. M. Sharir and P. K. Agarwal, Davenport-Schinzel Sequences and Their Geometric Applications, Cambridge University Press, Cambridge, 1995.

Received May 14, 2004, and in revised form November 16, 2004. Online publication March 30, 2005. 